\title{
$63-10$
}

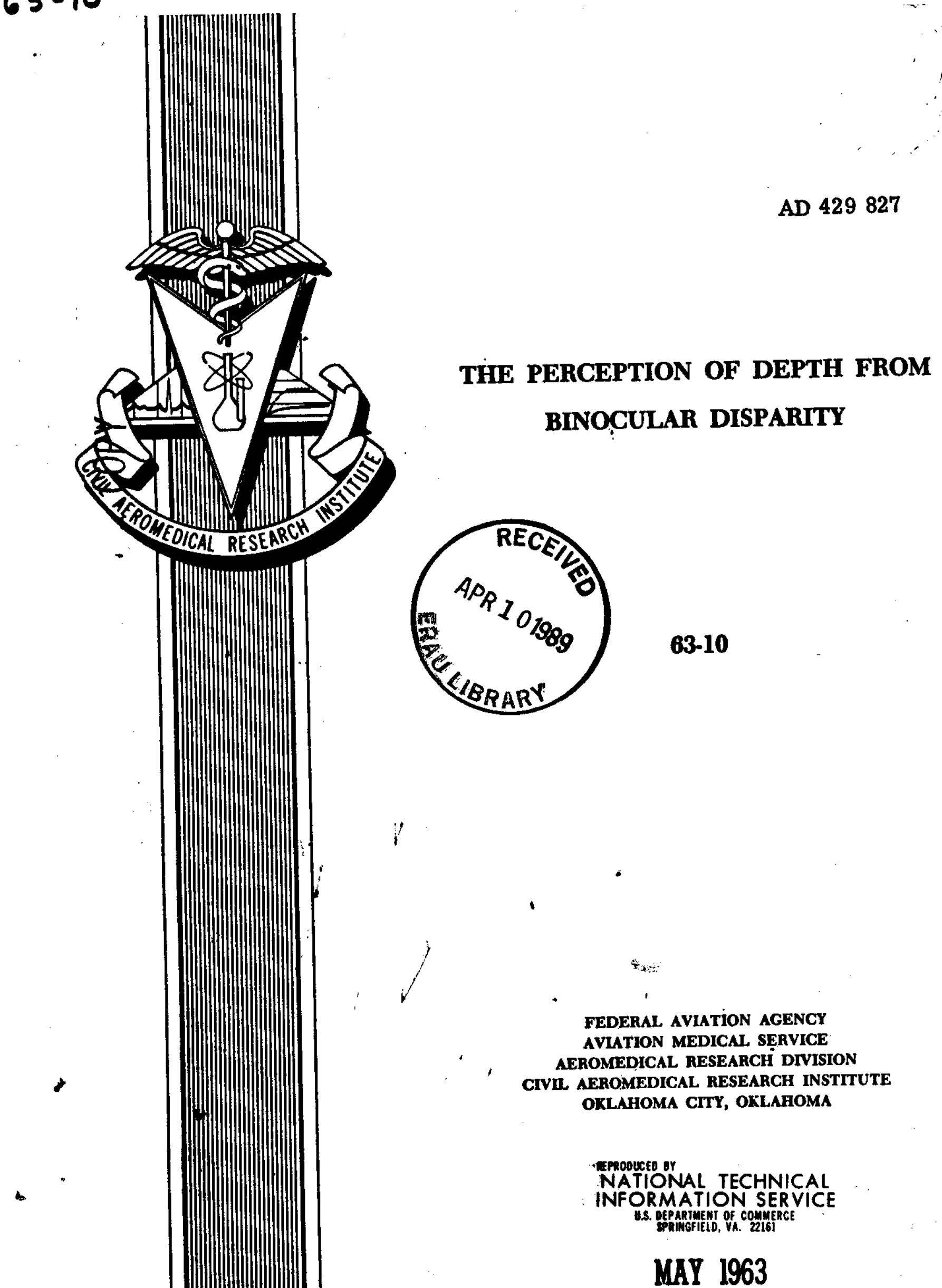




\section{THE PERCEPTION OF DEPTH FROM BINOCULAR DISPARITY}

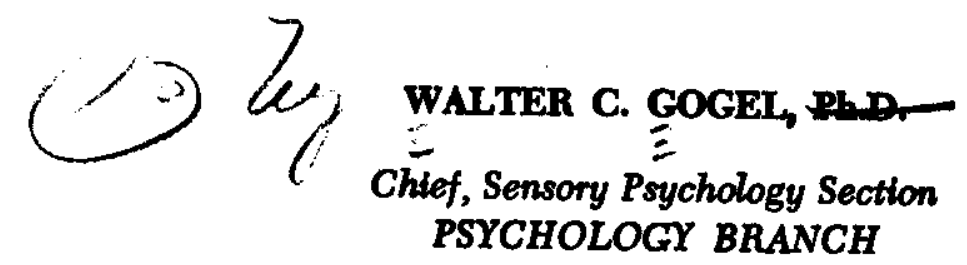

63-10

i

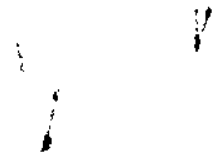

1

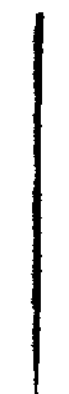

,

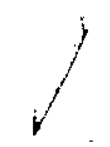

$$
+
$$

FEDERAL AVIATION AGENCY

AVIATION. MEDICAL SERVICE AEROMEDICAL RESEARCH DIVISION CIVIL AEROMEDICAL RESEARCH INSTITUTE OKLAFOMA CITY, OKTAHOMA

MAY 1963 


\title{
THE PERCEPTION OF DEPTH FROM BINOCULAR DISPARITY'
}

\author{
WALTER C. GOGEL, Ph.D.
}

One of the cue systems used in the perception of the depth between objects is the cue of binocular disparity. The purpose of the present study is to demonstrate the factor which determines the perceived depth interval which will result from the presence of a particular binocular disparity fot a particular/observer $(O)$. As has been pointed out (sed Von Kries [1925] for example), a constant binocular disparity will not necessarily be perceived by $O$ as producing the same depth interval at different distances from himself. It follows that the perceived extent associated with the binocular disparity is hot determined exclusively by the magnitude of the binocular disparity. Some factor which varies as a function of the distance
from $O$ must be involved in the process of translating the binocular disparity to a per- ceived depth extent. Two such factors which have been considered are (1) the convergence of the eyes, and (2) the perceived distance from $O$ of the objects producing the binocular disparity (see Fry, 1950; Ogle, 1953; Ogle, 1959; Von Kries, 1925). However, evidence is available agfinst each of these possibilities (Gogel, 1962c) and a different explanation will be used in the present study.

'The author wishes to thank Frank L. Agee, Jr., and Kerry C. Booth for their assistence in collocting and analyzing the data of this study. 
The hypothesis used in the present study to explain the perceived depth resulting from a binocular disparity is based upon the demonstrated ability of $O$ to equate the perceived depth from a binocular disparity to the perceived size of an adjacent frontoparallel extent (Gogel, 1958a; Gogel, 1958b; Gogel, 1960b). It is asserted that the perceived depth associated with a binocular disparity depends upon the perceived size associated with adjacent frontoparallel extents (Gogel, 1960a; Gogel, $1960 \mathrm{~b}$; Gogel, 1960c). This is an example of what has been termed the "adjacency principle" (Gogel, 1962c). The perceived depth interval which results from a particular binocular disparity is determined by the perceived frontoparallel size $\left(S^{\prime}\right)$ at the distance of the objects producing the binocular disparity. More specifically (Gogel, 1962c)',

$$
\Delta \mathrm{d}^{\prime}=\frac{\mathrm{s}^{\prime}}{\theta} \quad \frac{\Delta \alpha}{\mathrm{C}}
$$

where $\Delta \alpha$ is a small amount of binocular disparity, $\Delta \mathrm{d}^{\prime}$ is the perceived depth interval which results from $\Delta \alpha$, and $S^{\prime}$ is a perceived size of a frontoparallel extent whose retinal size (visual angle) is $\theta$. The term $C$ is a constant for a particular $O$. The ratio $S^{\prime} / \theta$ is the perceived size per unit of retinal size at the distance of the objects producing $\Delta \alpha$. As a ratio, $S^{\prime} / \theta$ is independent of any particular frontoparallel extent and can be applied to an entire frontoparallel plane.

It is evident that $S^{\prime} / \theta$ usually is not constant throughout the visual field. For example, if perfect frontoparallel size constancy' is present, for a constant value of $\theta, S^{\prime}$ and hence $S^{\prime} / \theta$ will increase linearly with the physical distance from $O$. It is clear, therefore; that according to Equation 1, a constant value of binocular disparity, will produce different perceived extents when it is perceived to be in portions of the visual field having different values of $S^{\prime} / \theta$. It is the purpose of the present experiment to test this point of view. Objects producing a constant value of binocular disparity will be made to appear in portions of a field-of-view having different values of $S^{\prime} / \theta$. The resulting perceived depth interval should increase as the value of $S^{\prime} / \theta$ increases.

\section{APPARATUS}

\section{The Binocular Stimult}

A perspective schematic drawing of the apparatus is given in Fig. 1. A binocular disparity was generated by binocularly viewing a configuration produced by two pairs of objects. One pair consisted of two luminous half-circles (Objects $e$ and f) and the other pair consisted of two luminous rectangles (Objects $g$ and $h$ ). The four objects $(e, f, g$, and $h$ ) were physically located to the right of $O$ (see Fig. 1 ). Light from these objects passed through a black tunnel and was reflected from a mirror into the eyes of $O$. The result was that $O$ perceived the objects to be in front of himself instead of at their actual locations. The perceived positions of Objects e, $f, g$, and $h$ are given in Fig. 1 as $e^{\prime}, f, g^{\prime}$, and $h^{\prime}$, respectively.

As is illustrated in Fig. 1, Objects e, $f, g$, and $h$ were formed by placing a diffused fluorescent source of light ( $94 \mathrm{ft}$. $-\mathrm{L}$.) behind appropriate apertures, (cf. Lichten \& Lurie 1950). Care was taken that no light from the portion of the apparatus producing Objects $e, f, g$, and $h$ was visible except that from the objects. Both Objects $\mathbf{g}$ and $\mathbf{h}$ (the luminous rectangles) were physically $277.2 \mathrm{~cm}$. from $O$ while both Objects $e$ and $f$ (the luminous half-circles) were physically $252.0 \mathrm{~cm}$. from $O$. The halfcircles were always higher in the visual field (by 30 minutes of visual angle) than the luminous rectangles. For the average interpupillary distance of the Os used in this study $(6.46 \mathrm{~cm}$.), the binocular disparity between the pair of luminous rectaritites and the pair of half-circles was, 477 seconds-of-arc. The height and width of Objects $g$ and $h$ subtended visual

\footnotetext{
Originally, (see Gogel, 1860b), the terms $\Delta \alpha^{\prime}$ and $\mathrm{E}$ were used in Equation 1 instead of the $\Delta d^{\prime}$ and $S^{\prime}$, respoctively, of the present study. The meanings associated with the former terms however are identical to those of the present study.
} 


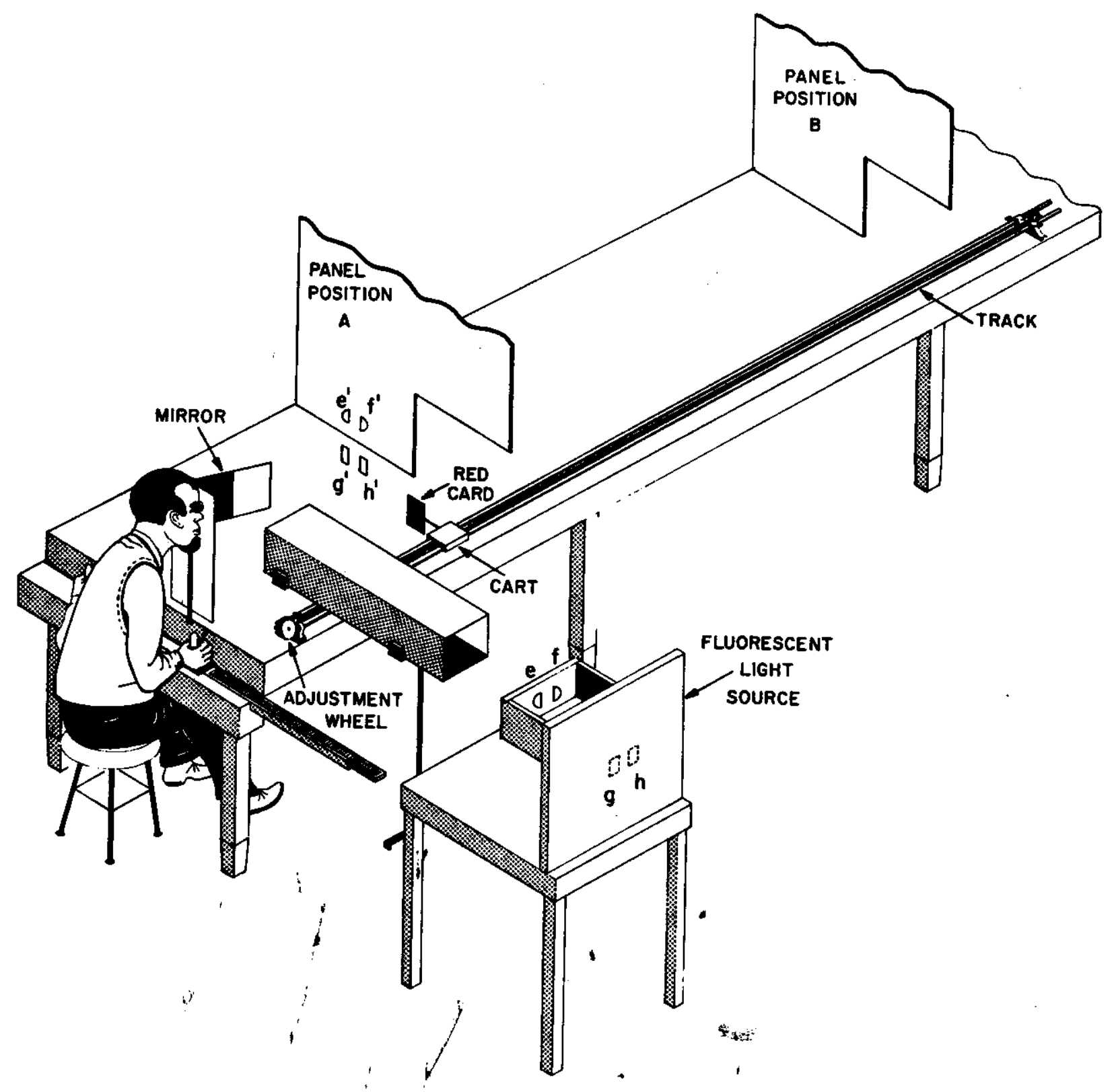

Ficonr 1. A schematic perspective drawing of the apparatus used in the study.

angles of 45 and 22.5 minutes-of-arc, respectively. The sizes of Objects e and $f$ were such as to subtend a visual area equal to that subtended by Objects $g$ and $h$. The horizontal

- separation between Objects e and $f$ or between Objects $g$ and $h$ was $2^{\circ} 57^{\prime}$ of visual angle.
The physical position of Objects $e, f, g$, and $h$ was constant throughout the study. Thus, both the convergence values of the eyes to the $\mathrm{Ob}$ jects $e, f, g$, and $h$ in the binocular configuration and the binocular disparities between these objects were invariant throughout this study. 


\section{The Alley}

As illustrated by Fig. 1, Objects $e^{\prime}, f^{\prime}, g^{\prime}$, and $h^{\prime}$ appeared to be located in an alley which extended away from $O$ in distance. The floor of the alley ( $48 \mathrm{ft}$. long and $4 \mathrm{ft}$. wide) consisted of a checkerboard pattern of light and dark grey rectangles ( 8 inches wide and 16 inches long). The alley was illuminated by a series of overhead fluorescent lights extending the length of the alley. The sides of the alley were formed on the right by white curtains and on the left by a wall covered with white cloth. The alley and all objects in the alley except Objects e, f, g, and $h$ were observed monocularly. This was accomplished by opaquing the back surface of that portion of the mirror which was in front of the left eye of $O$. The mirror was a first surface mirror which both reflected and transmitted light. This enabled $\boldsymbol{O}$ to view Objects $e, f, g$, and $h$ with both eyes while viewing the alley with his right eye only. Care was taken that the left eye of $O$ was in darkness except for Objects $e, f, g$, and $h$.

To remove extraneous light, the viewing position of $O$ was enclosed in black velveteen and a small black tube extended from the left eye of $O$ to the vicinity of the left half of the mirror. To eliminate the accommodative cue between objects in the visual field, $O$ viewed the entire scene through two pin hole apertures (.05 cm. in diameter) located very close to his eyes. When a small black curtain in front of the viewing position was lowered, the view to the monocularly observed alley was cut off without disturbing the ability to see the binocular stimuli. An adjustable chin rest was located at the viewing position of $O$ and a light adaptation surface, (51 ft. - L.),' which could be turned on or off by the experimenter (EY) was located to the left of the viewing position.

To make the constant binocular configuration appear in different portions of the monocularly observed alley at different times, a monocularly observed panel consisting of diagonal black and grey stripes was vertically presented at either 182.9 or $548.6 \mathrm{~cm}$. from $O$ in the alley. The two alternate positions of the panel are illustrated as A and B of Fig. 1. It was anticipated that the binocular configuration would appear to $O$ to be closer to himself (in a less distant portion of the monocularly observed alley) when the panel was in position $A$ rather than in position B. Since some amount of frontoparallel size constancy would be expected to occur in the alley, the different distance positions in the alley would be expected to have different values of $S^{\prime} / \theta$. The process by which the panel would make the binocular configuration appear to change in distance position in the monocularly observed alley is called the "equidistance tendency" (Gogel, 1956). This is the tendency for objects to appear equidistant unless restricted by the presence of effective depth cues. It is clear that, except for the equidistance tendency, there was no reason why the binocular configuration would appear at one distance rather than another in the alley. The only factor other than the equidistance tendency available for localizing the binocular configuration in apparent distance was the convergence of the eyes required for binocular fusion. But, it has been demonstrated that this cue is usually ineffective in establishing a perceived distance (Gogel, 1962b). Thus, the equidistance tendency would be expected to be effective between the binocularly observed objects and the monocularly observed panel with the result that the binocular configuration would appear to be at the distance vicinity of the panel.

\section{Measuremente of Apparent Size and Diutance}

Three types of pśychological measurements were required in this study. It was necessary to determine (1) that the change in the position of the panel was effective in inducing a change in the apparent position of the binocular configuration (Objects $e^{\prime}, f, g^{\prime}$, and $h^{\prime}$ ), (2) that concomitant with the change in apparent position of the binocular configuration, the value of $S^{\prime} / \theta$ associated with the frontoparallel extents of the binocular configuration ako changed, and (3) that the apparent depth within the binocular configuration changed in agreement with the change in $S^{\prime} / \theta$.

The measurement of the apparent depth position of Objects $e^{\prime}, \boldsymbol{P}, \boldsymbol{g}$, and $h^{\prime}$ was accomplished by using the track and cart (see Fig. 1). 
Attached to the cart by means of a black rod was a red card $(10.1 \mathrm{~cm}$. wide by $12.7 \mathrm{~cm}$. high). presented vertically on the floor of the alley. The bottom of the red card always made contact with the floor of the alley. The red card was monocularly observed (with the right eye) and its apparent distance position in the alley was clearly seen by the interposition of its bottom edge with the alley floor. By turning a knob with his right hand, $O$ could move the cart along the track and consequently could move the red card in distance along the alley to the apparent depth position of Objects e, $f$, $g$, or $h$ as required.

To measure the values of $S^{\prime}$ in the binocular configuration, a distance indicator using the kinesthetic modality was located at the position of $O$. The device consisted of two vertical rods which $O$ could grasp, one in each hand. The left rod was stationary while the right rod could be moved laterially until the separation between the rods seemed to $O$ to be the same as a designated frontoparallel distance (width) in the binocular configuration. The hand adjustment apparatus was not visible to $O$ during the width adjustments. An attached scale permitted the experimenter (E) to measure the lateral distance $\left(\mathrm{S}^{\prime}\right)$ which $O$ adjusted between the rods. A similar device has been used to measure perceived size in previous studies (Gogel, 1962a; Gogel, 1962b). From the visual angle $(\theta)$ of the width being perceptually duplicated and the obtained adjustment $\left(S^{\prime}\right)$, the ratio $S^{\prime} / \theta$ could be computed.

The apparent depth within the binocular configuration (the apparent depth from the rectangles to the half-circles) was also measured by means of the hand adjustment apparatus. The $O_{s}$ kinesthetically adjusted the laterial distance between the rods uxtil it appeared equal to the designated depth interval in the binocular configuration.

\section{OBSERVERS}

Ten men end six women were used as $O$ s. Each $O$ had a stereoscopic acuity of at least 18 seconds-of-arc as measured with the Keystone Multi-Stereo Test. As a consequence of a preliminary test, several potential $O$ s were not used in the experiment because they saw some portion of the binocular configuration imbedded in the surface of the panel.

\section{PROCEDURE}

To determine that $O$ saw the alley as extending away from himself in distance, a white (monocularly observed) target, $7.6 \mathrm{~cm}$. wide and $20.3 \mathrm{~cm}$. high, was presented vertically on the floor of the alley at randomly presented distances of either $140,235,330,425$, or $520 \mathrm{~cm}$. from $O$. Each $O$ adjusted the red card to apparent equidistance with the white target at each of the physical distances, and also made verbal estimates in feet of the distance of the white target from himself. During this procedure, the striped panel was located at its far position. Following these adjustments, the white target was removed and the front curtain was lowered, eliminating the view of the alley. The binocular stimuli were turned on and $O$ checked to determine that he saw all four objects of the binocular stimulus (e, $f, g$, and $h$ ) with each eye. The front curtain was raised and $O$ was asked to adjust the lateral distance between the inner edges of the rods of the hand adjustment apparatus until it seemed to be the same physical size as the extent between either:

(1) the inner edges of the half-circles (a judgmẹnt of width, $S^{\prime}$ ') or;

(2) the inner edges of the rectangles (a judgment of width, $S^{\prime}$ ) or;

(3) the depth between the left half-circle and the left rectangle (a judgment of depth, $\left.d^{\prime}{ }^{\prime}\right)$ or;

(4) the depth between the right half-circle and the right rectangle (a judgment of depth, $\mathrm{d}_{\mathrm{n}}$ ).

For a particular position of the panel and a particular $O$, all judgements of width (or depth) were completed before proceeding to the judgments of depth (or width).

Thus, each $O$ used the hand adjustment apparatus to make two width and two depth judgments for each of the two positions of the panel. Each $O$ repeated each width or depth judgment three times. The starting position 


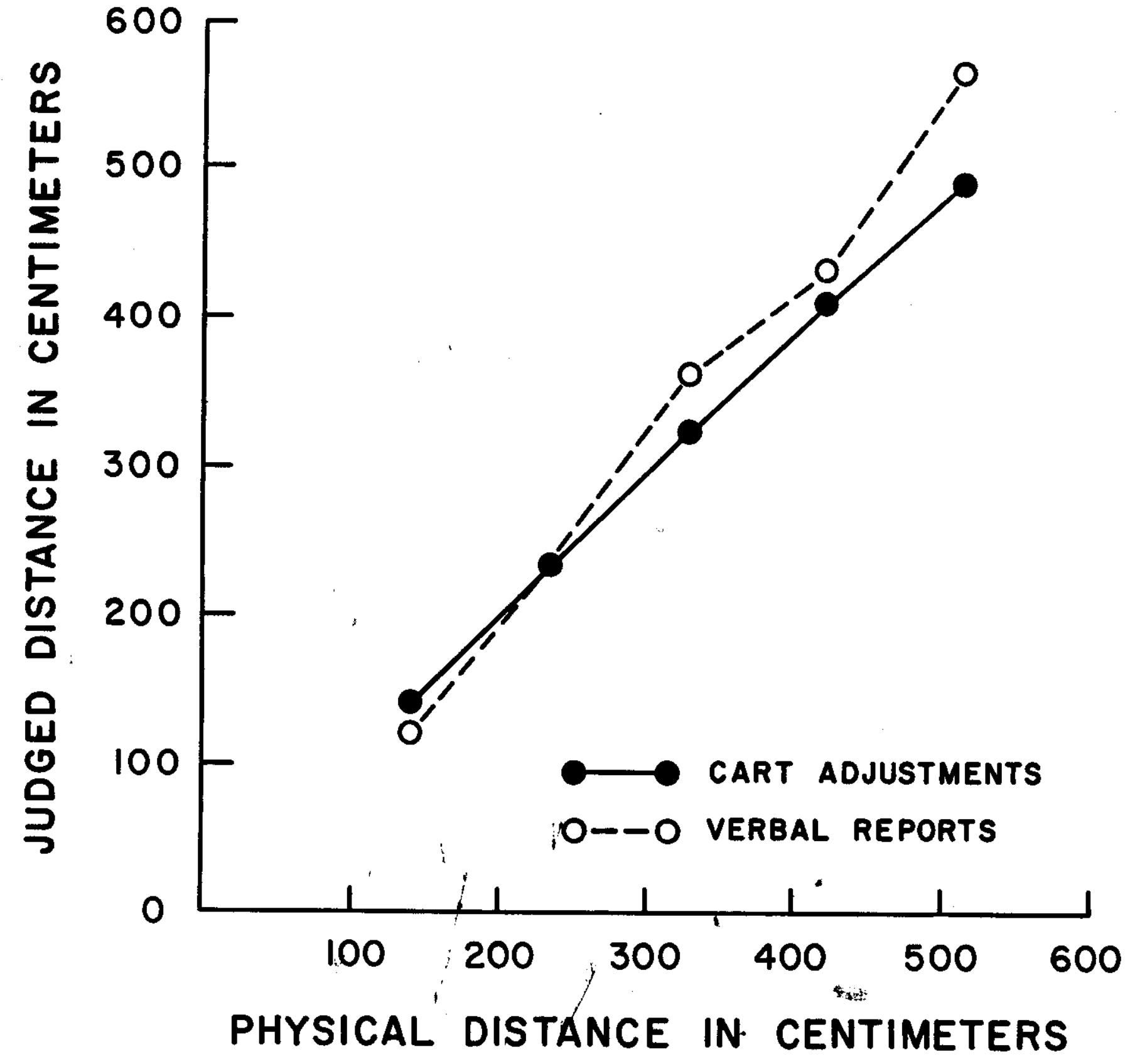

Frevis 2. The relation between behavioral and physical distance to the alley.

for the hands for these adjustments (hands together or hands far apart), was systematically varied. Following each set of three width or depth judgements, $O$ adjusted the red card to the distance of each of the two binocular objectsinvolved in the particular judgment, verbally estimated in feet the distance to each binocular object, and turned to look at the light adaption surface which was turned on by $E$ for approximately $10 \mathrm{sec}$. After completing the width and depth judgments for one position of the striped panel, $O$ adjusted the red card to the distance of the panel, and verbally estimated this distance from himself in feet. Following this, the striped panel was moved to the second distance and the judgments were 
repeated. With half the $O s$, the panel was presented first at the near position and then at the far position. With the remaining $O s$, the order of presentation was reversed.

\section{RESULTS}

Figure 2 contains the average results expressed in centimeters obtained both from the adjustment of the red card to the apparent distances of the white target and from the verbal reports of the distances of the white target. It is clear from Fig. 2 that the alley appeared to extend away from $O$ in distance and that increasing values of cart adjustment or verbal reports represent increasing values of perceived distance.

The mean and median results in centimeters for the hand adjustments are given in Table 1 . The measurements of perceived depth between the left and right pairs of binocular objects have been averaged in the d' data of Table 1 . For two $O s$, the perceived depth within the binocular configuration was greater than could be measured with the hand adjustment apparatus. These two $O s$ were asked to perceptually duplicate only $1 / 3$ of the magnitude of each extent within the binocular configuration and, therefore, the results from these Os were multiplied by three to arrive at the values which would be expected to occur if these $O s$ had been able to make the apparent duplications directly. Some of the distributions of hand adjustments are skewed positively. For each position of the panel, the width adjustments in Table $l$ are separated into the adjustments measuring the perceived width between the half-circles $\left(S_{*}^{\prime}\right)$ and the adjustments measuring the perceived width between the rectangles $\left(S_{+}^{\prime}\right)$. The values of width adjustinents were transformed into $S^{\prime} / \theta$ by dividing each value of $S^{\prime}$ in Table 1 by the constant value of $\theta$ $\left(2^{\circ} 57^{\prime}\right)$. The $S^{\prime} / \theta$ value for the $548.6 \mathrm{~cm}$. position of the panel is 1.30 and $1.53 \mathrm{~cm}$. per degree for the width between the half-circles and the rectangles, respectively. For the $182.9 \mathrm{~cm}$. position of the panel; $S^{\prime} / \theta$ is 1.07 and $1.18 \mathrm{~cm}$. per degree for the width between the halfcircles and the rectangles, respectively. The six distributions of scores whose means are given in Table 1 were converted to common logarithms. The differences between the average logarithms'paralleling the mean differences listed in Table 1 all proved to be statistically significant using the $t$ test at the .01 level. It is clear that some amount of frontoparallel size constancy was present in this experiment. Also, Table 1 indicates that the perceived depth within the configuration of binocular objects increased as the value of $S^{\prime} / \theta$ increased. The change in depth between the rectangles and the half-circles occurred in spite of both the binocular disparities within the configuration and the

\section{TABLE' 1}

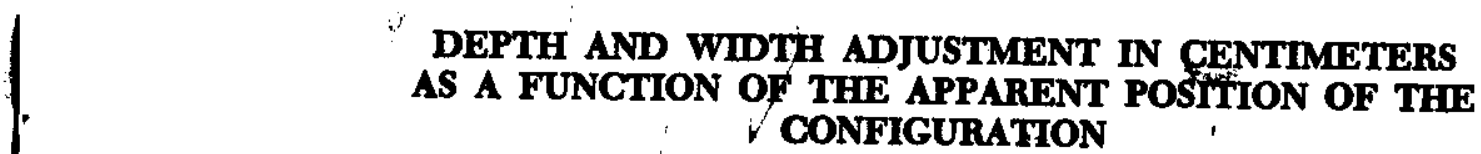

DEPTH AND WIDTH ADJUSTMENT IN CENTMMETERS CONFIGURATION

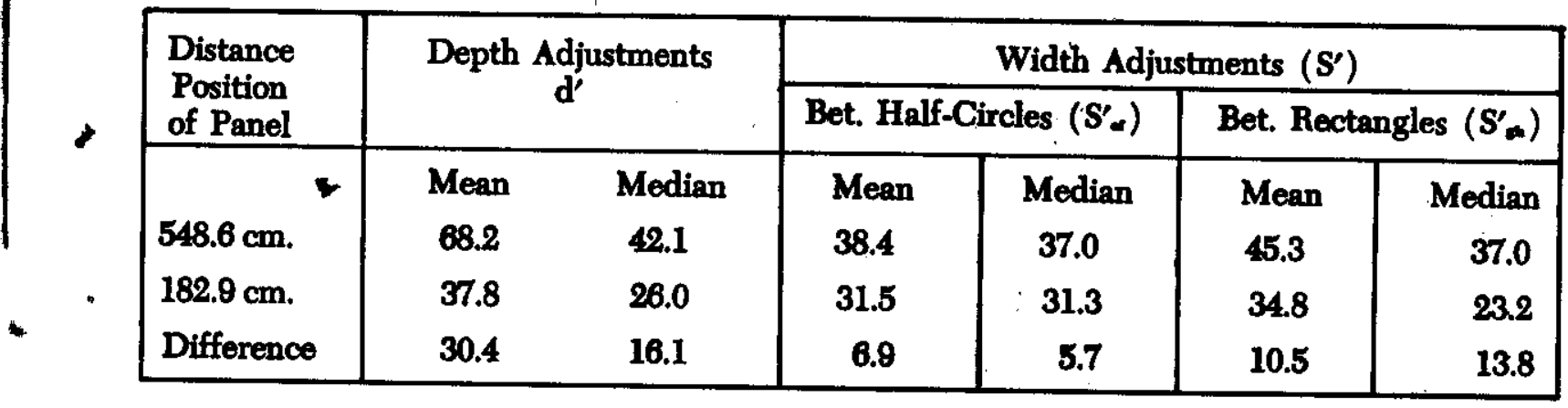


convergences to the configuration remaining constant throughout the experiment.

The mean and median results (in centimeters) from the cart adjustments to, and the verbal reports of, the apparent distance of $\mathrm{Ob}$ jects $e, f, g$, and $h$ are given in Table 2. The differences between the median and mean verbal reports in Table 2 are mostly due to the results from one $O$. From Table 2, the binocular configuration appeared to be more distant when the panel was more distant. For all Os except one, both the verbal reports of, and the cart adjustments to, Objects $e^{\prime}, f^{\prime}, g^{\prime}$, and $h^{\prime}$ always were greater for the far rather than for the near position of the panel. In agreement with the results from the hand adjustments, both the verbal reports and cart adjustments of Table 2 indicate that the apparent depth within the binocular configuration was greater when the binocular configuration appeared in a more distant portion of the alley.

The average cart adjustment to the apparent distance of the striped panel was 535.1 and $183.5 \mathrm{~cm}$. for the greater and smaller distances of the panel from $O$, respectively, with corresponding verbal reports (converted to centimeters) of 664.5 and $190.0 \mathrm{~cm}$. The apparent position of the panel clearly changed as its physical distance was changed. Also, comparing the apparent panel positions with the results shown in Table 2 , it is clear that with these Os, for both positions of the panel, the most distant portion of the binocular configuration appeared not on the surface of the panel but, instead, the entire binocular configuration appeared to be suspended in the visual field in front of the panel.

\section{DISCUSSION}

As discussed elsewhere (Gogel, 1960a; Gogel, $1960 \mathrm{~b})$, Equation 1 should be applied only to small values of binocular disparity. For larger values of binocular disparity, such as were used in the present experiment, the perceived depth $d^{\prime}$ should be expressed as the sum of a series of smaller perceived extents. The equation resulting from such a summation process and using Equation 1 as a basis is

$$
\mathrm{d}^{\prime}=\frac{1}{C} \int^{\cdot} \frac{\mathrm{s}^{\prime}}{\theta} \mathrm{d} \alpha
$$

TAbre 2

AVERAGE CART ADJUSTMENTS TO, AND VERBAL "REPORTS OF, THE APPARENT POSTION OF OBJECTS e, f, g, AND $h$

\begin{tabular}{|c|c|c|c|c|c|c|c|c|}
\hline \multirow{4}{*}{$\begin{array}{c}\text { Distance } \\
\text { Position } \\
\text { of } \\
\text { Panel }\end{array}$} & \multicolumn{8}{|c|}{ Apparent Distance of Objects } \\
\hline & \multicolumn{8}{|c|}{ Means } \\
\hline & \multicolumn{2}{|c|}{ Object e } & \multicolumn{2}{|c|}{ Object'f } & \multicolumn{2}{|c|}{ Object $\mathbf{g}$} & \multicolumn{2}{|c|}{ Object h } \\
\hline & $\begin{array}{c}\text { Cart } \\
\text { Adjustment }\end{array}$ & $\begin{array}{l}\text { Verbal } \\
\text { Report }\end{array}$ & $\begin{array}{c}\text { Cart } \\
\text { Adjustment }\end{array}$ & \begin{tabular}{|l|} 
Verbal \\
Report'
\end{tabular} & $\begin{array}{c}\text { Cart } \\
\text { Adjustment }\end{array}$ & $\begin{array}{l}\text { Verbal } \\
\text { Report }\end{array}$ & $\begin{array}{c}\text { Cart } \\
\text { Adjustment }\end{array}$ & $\begin{array}{l}\text { Verbal } \\
\text { Report }\end{array}$ \\
\hline \multirow{3}{*}{$\begin{array}{l}548.6 \mathrm{~cm} . \\
182.9 \mathrm{~cm} .\end{array}$} & 314.7 & 304.2 & 295.8 & 288.9 & 398.8 & 428.4 & 392.3 & 417.5 \\
\hline & 151.6 & 133.9 & 152.2 & 136.2 & 173.7 & 177.7 & 176.5 & 187.2 \\
\hline & \multicolumn{8}{|c|}{ Medians } \\
\hline $548.6 \mathrm{~cm}$. & 338.4 & 221.0 & 322.6 & 213.4 & 493.1 & 301.0 & 403.2 & 301.0 \\
\hline $182.9 \mathrm{~cm}$. & 153.7 & 118.1 & 158.0 & 118.1 & 176.3 & 160.0 & 178.8 & 160.0 \\
\hline
\end{tabular}


In Equation 2, two factors in addition to the individual constant $\mathrm{C}$ are important in the determination of $d^{\prime}$. One factor is the value of binocular disparity $\alpha_{0}-\alpha_{t}$ where $\alpha_{0}$ and $\alpha_{1}$ are the convergence values of the eyes to the near object (e) and the far object (f) producing the disparity. The other factor is the variation in $S^{\prime} / \theta$ between the convergence limits $\alpha_{t}$ and $\alpha_{t}$ of the eyes. Equation 2 is designed to apply to situations in which the convergence value $\left(\alpha_{e}\right)$ to the nearest binocular object in the binocular configuration specifies the apparent distance position of this object in the field-ofview. This does not imply that the convergence of the eyes is considered to be an effective distance cue. An object in a binocularly observed field will appear at the distance of that object in the field with respect to which it has zero amount of binocular disparity. Geometrically, this is equivalent to stating that the two binocular objects will appear at the same distance when the convergence value of one equals that of the other. In the present study, the visual field was monocularly observed except for the binocular configuration, and only the equidistance tendency was available to perceptually localize the binocular configuration in depth. Therefore, Equation 2 is not in a form which can be applied directly to the present study. A consideration of Equation 2 does serve to illustrate, however, that Equation 1, while valid for small depth intervals, can be expected to predict the direction but not necessarily the magnitjlde of the changes in perceived depth ( $\left.d^{\prime}\right)$ which occurred as a consequence of the changes in $S^{\prime} / \theta$ in the present study.

The present experiment demonstrates that the perceived depth resulting from a constant binocular disparity can' differ depending upon the portion of the field-of-view in which the binocular configuration appears. This phenomenon is attributed to the different values of $S^{\prime} / \theta$ in different parts of the visual field. However, both the perceived absolute distance of the binscular configuration and the $S^{\prime} / \theta$ values within the configuration changed for the two positions of the panel. But, from previous evidence (Gogel, 1960a), it is concluded that the change in the perceived size per unit of frontoparallel size $\left(S^{\prime} / \theta\right)$, not the change in perceived absolute distance, is the significant variable.

The present study asserts the importance of so-called "monocular cues" upon the depth perception resulting from binocular cues. Many cue systems affect $S^{\prime}$ and, through $S^{\prime} / \theta$, affect the perception of depth from binocular disparity. Thus, the monocular cues, to the extent that they determine $S^{\prime}$, also determine the perceived depth associated with a particular binocular disparity. This statement is not equivalent to the truism that a variety of cue systems contributes to the perception of depth. The assertion is that the perceived depth from binocular disparity is not predictable unless the perceived size per visual angle of frontoparallel size $\left(S^{\prime} / \theta\right)$ in the depth vicinity of the disparity is known. Except in the case of zero amount of size constancy, the value of $S^{\prime} / \theta$ will differ for different distances in the visual field. Unless the values of $S^{\prime} / \theta$ are known, the perceived depth from a binocular disparity in any specified part of the visual field cannot be predicted. It is clear from the present study that the amount of size constancy present and the magnitude of the perceived depth between binocular objects are interrelated phenomena and a theory of binocular vision is incomplete unless it provides for this interrelation.

\section{REFERENCES}

Fry, G. A. Visual perception of space. Amer. J. Optom., $1050,27,531-553$.

Gogel, W. C. The tendency to see objects as equidistant and its inverse relation to lateral separation. 'Prychol. Monogr., 1956, 70, No. 4, (whole No. 411).

Gogel, W. C. An observer constant in the perception of stereoscopic depth. U.S. Army Med. Res. Lab. Rep. 1958, Fort Knox, No.sto. (a).

Ģogel, W. C., The effect of convergence and angular size upon the computed value of a hypothetical observer constant in binocular vision. U.S. Army Med. Res. Lab. Rep. 1958, Fort Knox, No. 372.(b).

Gogel, W. C. Perceived frontal size as a determiner of perceived binocular depth. J. Prychol., 1960, 50, 119-131. (a).

Gogel, W. C. The perception of shape from binocular disparity cues. J. Psychol., 1860, 50, 179-192.(b).

Gogel, W. C. The perception of a depth interval with binocular dispartty cues. J. Prychol., 1960, 50, 257-269.(c).

Gogel, W. C. Convergence as a determiner of percelved absolute size. J. Psychol., 1962, 53, 91-104.(a). 
Gogel, W. C. The effect of convergence on percetved size and distance. J. Psychol., 1962, 53, 475-489.(b).

Gogel, W. C. The visual perception of size and distance. Civ. Aeromed. Res. Lab. Rep., 1962, Oklahoma City, No. 62-15.(c).

Lichten, W. and Lurle, S. A new technique for the etudy of perceived stze. Amor. J. Paychal, 1050, 63, 280-282.
Ogle, K. N. Precision and validity of stemeoscopic depth perception from double images. J. Opt. Sac. Amer., 1953, 43, 806-813.

Ogle, K. N. Theory of steroscoptic vision. In S. Koch (Ed.), Prychology: A sudy of a eclence, Vol. I, Now Yort: McGraw-Hill, 1850, 302-394.

Von Kries, J. Notes on perception of depth. In H. V. Helmholtz's Treatios on Physiological Optics, Vol. III, (I. P.C. Southall, Ed.) Menashe, Wisconsin: Banta, 1925, $362-100$,

$$
\text { sic: }
$$

